Check for updates

Cite this: RSC Adv., 2019, 9, 39834

\title{
Persea americana seed extract mediated gold nanoparticles for mercury(II)/iron(III) sensing, 4- nitrophenol reduction, and organic dye degradation $\uparrow$
}

\begin{abstract}
Naveen Kumar Reddy Bogireddy (D) * and Vivechana Agarwal (DD *
In this work, Persea americana (Avocado) seed extract mediated systematically optimized synthesis has been employed for the formation of small sized gold nanoparticles (Av-AuNPs) at different pH values. The size, shape and crystallinity of the as-prepared AuNPs have been studied using transmission electron microscopy and X-ray diffraction. The nanoparticles were found to be selective towards mercury(I) upon the prior or subsequent addition of iron(III), revealing a blue shift and an enhancement of the characteristic surface plasmon resonance (at $519 \mathrm{~nm}$ ). Similar absorbance based selectivity has been observed towards Fe(III) in the presence of $\mathrm{Hg}\left({ }_{1}\right)$. The high sensitivity and selectivity of Av-AuNPs towards $\mathrm{Hg}\left({ }_{1}\right)$ and $\mathrm{Fe}(\mathrm{II})$ has been attributed to the formation of core-shell structures. From the UV-visible spectroscopic measurements, the limits of detection for $\mathrm{Hg}(॥)$ and $\mathrm{Fe}(\mathrm{II})$ are found to be $50 \mathrm{nM}$ and $30 \mathrm{nM}$ (around one order of magnitude less than the Environment Protection Agency limit of $0.7 \mu \mathrm{M}$ for Fe(III) in drinking water) respectively, with an excellent linear dependence over a wide range of concentrations. Additionally, as-prepared Av-AuNPs have been demonstrated to be efficient in the reduction of organic pollutant 4-nitrophenol to 4-aminophenol and degradation of some organic dyes, such as Methylene Blue, Direct blue, Rhodamine 6G, Bromophenol blue and methyl orange. The use of the proposed Av-AuNPs for sensing and green catalysis can form the basis of high-performance analytical assays, effective multiplexed intracellular sensors, and sophisticated and sustainable probes/ catalysts.
\end{abstract}

Received 9th October 2019

Accepted 25th November 2019

DOI: $10.1039 / c 9 r a 08233 f$

rsc.li/rsc-advances

\section{Introduction}

The presence of an excessive amount of heavy metal ions in water is a vital problem for the environment, whereas the consumption of contaminated water causes severe health problems. Heavy metal ions commonly found in water, include $\mathrm{Fe}(\mathrm{II}) / \mathrm{Fe}(\mathrm{III}), \mathrm{Hg}$ (II), $\mathrm{As}$ (III), $\mathrm{Ni}$ (II), $\mathrm{Pb}$ (II), $\mathrm{Cu}$ (II) etc, and some of them are among the constituent elements of the earths' crust., ${ }^{1,2}$ Human activities, such as agricultural and industrial applications of metal compounds, are major causes of surface as well as ground water pollution. ${ }^{1,2}$

Mercury(II) and iron(III) are among the toxic ions in the group of heavy metals and their excess intake has been reported to cause serious health problems (nervous system problem, skin cancer, kidney disease, etc.). The permissible limit of mercury and iron present in water is $4 \mathrm{nM}$ and $0.7 \mu \mathrm{M}$ respectively. ${ }^{1,3,4}$ Due to the growing applications of heavy metal ions at the

Centro de Investigación en Ingeniería y Ciencias Aplicadas, UAEM, Av. Univ. 1001, Col. Chamilpa, Cuernavaca, Mexico.E-mail: nsbogireddy@gmail.com; vagarwal@uaem. $m x$

$\dagger$ Electronic supplementary information (ESI) available. See DOI: 10.1039/c9ra08233f industrial level, aquatic pollution has increased exponentially in recent years. As such, monitoring of heavy metal ions in contaminated water has become challenging among the scientific community. To detect heavy metal ions in contaminated water, there are many conventional but expensive, complicated and time-consuming ${ }^{5,6}$ techniques, such as inductively coupled plasma mass spectrometry, atomic fluorescence spectrometry, atomic absorption spectrometry etc.

Toward sensing of heavy metal ions in aqueous solution, surface plasmon resonance (SPR) based metal nanoparticles (MNPs: gold and silver NPs) is found to be a promising option. ${ }^{7,8}$ MNPs are basically different from the corresponding bulk materials due to their size, shape and surrounding dielectric environment. The unique opto-electro-chemical properties of MNPs, different ways for its application in sensing and remediation field have been demonstrated..$^{7-17}$ It is worth mentioning that most of the researchers have reported the detection of heavy metal ions based on colorimetric approach due to simplicity and cost effectiveness where the outcome can be observed by the naked eye., ${ }^{910}$ Although they are widely used, we still need to study an eco-friendly approach for sensing of heavy metal ions. ${ }^{7,8,18-22}$ To overcome these drawbacks, here we propose an alternative 
sensing probe with avocado seed extract. In this report, we have used a green reducing cum stabilizing agent in the form of avocado seed extract for the preparation of eco-friendly Av-AuNPs sensing probe towards the monitoring of heavy metal ions in aqueous medium. An additional motivation behind the preparation of avocado seed extract mediated Av-AuNPs is their low toxicity, eco-friendly processing and biocompatibility. The synthesized sensing probes have been tested for different concentrations of heavy metal ions and found to be selective towards mercury(II) and iron(III). Besides, the sensitivity and selectivity of the proposed sensing probe towards certain metal ions, it has been demonstrated to be useful for the degradation of dyes and reduction of 4-nitrophenol.

\section{Experimental}

\section{Materials and methods}

Analytical grade mercury chloride, iron chloride, iron sulfide, copper chloride, copper sulfide, arsenic, nickel sulfide and cobalt chloride, chloroauric acid $\left(\mathrm{HAuCl}_{4} \cdot 3 \mathrm{H}_{2} \mathrm{O}\right)$ were obtained from Sigma Aldrich and used without further purification. Avocado seeds (Av) were collected from local market in cuernavaca, Mexico. Millipore water was utilized throughout the synthesis process.

\section{Material characterization}

UV-vis optical absorption spectra of the Av extract, colloidal AvAuNPs and metal ion sensing experiments were recorded in a dual beam PerkinElmer Lambda 950 spectrophotometer. The $\mathrm{X}$-ray diffraction studies of the Au nanoparticles was carried out in a Bruker D8 Advance eco diffractometer using its $\mathrm{CuK} \alpha(\lambda=$ $1.5406 \AA$ A ) radiation. The X-ray diffraction spectra was recorded in the $2 \theta$ range $10-90^{\circ}$, with a scanning rate and step size of $40 \mathrm{~min}^{-1}$ and 0.02 respectively. The morphology, size, and nanostructure of the prepared Av-AuNPs were inspected in a JEOL JEM 2100 transmission electron microscope (TEM) operating at $70 \mathrm{kV}$ accelerating voltage with $200 \mathrm{k} \times$ magnification. Dynamic light scattering (Malvern zetasizer, model nano ZS) technique was used to determine the hydrodynamic size distribution of the Av-AuNPs. To investigate the surface characteristics of the synthesized AuNPs, their room temperature ATR-Fourier transform infrared (FT-IR) spectra were recorded in a Varian 660-IR FT-IR spectrophotometer.

\section{Preparation of avocado seed extract}

Avocado seeds, collected from the local market were grinded to fine powder, and stored in inert conditions. For the preparation of avocado extract, $1 \mathrm{~g}$ of its finely grinded powder was taken in a beaker containing $100 \mathrm{~mL}$ of distilled water and kept in temperature-controlled heating mantle at $70{ }^{\circ} \mathrm{C}$ for $40 \mathrm{~min}$. Then the extract was filtered using a cellulose nitrate filter paper.

\section{Effect of reaction parameters on the synthesis of AuNPs}

Reaction parameters including reaction conditions, $\mathrm{HAuCl}_{4}$ concentration, plant extract volumetric ratio and $\mathrm{pH}$ have been taken into consideration for determining different possibilities for efficient preparation of AuNPs. A certain volume of gold ion solution $\left(\mathrm{HAuCl}_{4} \cdot 3 \mathrm{H}_{2} \mathrm{O} ; 1 \mathrm{mM}\right.$ ) was added to freshly prepared avocado extract in $1: 2$ volumetric ratio and kept under ambient conditions for about $28 \mathrm{~min}$. Gradual reduction of $\mathrm{Au}$ ions and the formation of AuNPs could be perceived visually though gradual color change of the reaction mixture, which turned from pale yellow to ruby red. Formation of AuNPs was further confirmed by UV-vis spectrophotometry. For the fabrication of AuNPs of different sizes, the same procedure was repeated, only by varying the $\mathrm{pH}$ of the Avocado seed extract by adding buffer solutions to maintain various $\mathrm{pH}$ values.

\section{Metal ion sensing}

The principal absorption band of as-prepared Av-AuNPs (around $519 \mathrm{~nm}$ ) at ambient conditions, was monitored for the absorbance based optical detection of $\mathrm{Hg}$ (II) and $\mathrm{Fe}$ (III). It was noticed that under the used experimental conditions, detection of $\mathrm{Hg}$ (II) and $\mathrm{Fe}$ (III) ions individually through AuNPs, is not possible. The detection of $\mathrm{Hg}$ (II)/Fe(III) by the subsequent addition $\mathrm{Fe}$ (III) $/ \mathrm{Hg}$ (II) into AuNPs was studied by monitoring the temporal evolution of the intensity of characteristic absorption bands of AuNPs (around $519 \mathrm{~nm}$ ) in the reaction mixture, along with an observable SPR blue shift during the experiment.

\section{Organic pollutant and dyes reduction/degradation}

As-prepared Av-AuNPs were additionally tested for the catalytic reduction/degradation of industrial organic contaminants (IOCs) in the presence of sodium borohydride $\left(\mathrm{NaBH}_{4}\right)$. As a first step (control reaction), IOCs $(1 \mathrm{mM})$ and the reducing agent $\mathrm{NaBH}_{4}(10 \mathrm{mM})$ were mixed in the absence of catalyst and the solution was left under observation for a long time. For catalytic reduction of IOCs, Av-AuNPs $\left(30 \mu \mathrm{g} \mathrm{mL}{ }^{-1}\right)$ were added to a similar solution containing intermediate form of IOCs. The catalytic degradation of IOCs was monitored by recording UVvisible absorbance spectra at regular time intervals.

\section{Results and discussion}

In order to obtain small sized Au NPs, the Au salt and AS extract concentrations were varied for a fixed concentration of AS extract and Au salt respectively. In general, AS extract was used as a green reducing-cum-stabilizing agent to prepare AuNPs with different SPR peaks by controlling reaction parameters/ conditions. While the aqueous AS extract is seen to be an effective reducing and stabilizing agent for the growth of $\mathrm{Au}$ nanoparticles, the reaction parameters of the synthesis processes have been found to play an important role in controlling the SPR absorption peak of the Au nanoparticles. Fig. S1 (ESI $\dagger$ ) presents the absorption spectra of the colloidal Au nanoparticles sythesized at different Au salt and AS extract concentrations with fixed final volume of the solution (Au salt : CA extract: $1: 2$ ), of the reaction mixtures (Fig. S1a $\dagger$ ). Formation of small Au nanoparticles is clear from the appearance of characteristic surface plasmon resonance (LSPR) bands in respective absorption spectra. Although the position of SPR 
band in the fabricated samples (by increasing the concentration of AS extract) showed a blue shift in the absorbance wavelength from 0.1 to $1 \mathrm{~g} / 100 \mathrm{~mL}$, observation of a relatively broader peak from 2 to $4 \mathrm{~g} / 100 \mathrm{~mL}$ is attributed to the formation of bigger sized AuNPs. On the other hand it is found to vary rather systematically while increasing the concentration of $\mathrm{Au}$ salt (Fig. S1a $\dagger$ ) at a fixed AS Extract concentration.

Fig. S2a $\uparrow$ shows real time monitoring of Au NPs' formation process using UV-visible absorption spectroscopy. Although before the addition of AS extract (Fig. S2a; $\dagger$ bottom black line) no absorption peak was observed with $\mathrm{Au}^{3+}$ ion solution, the addition of AS extract reveals a gradual increment in the absorption intensity with blue shift in the wavelength from 536 to $530 \mathrm{~nm}$ in a time span of $28 \mathrm{~min}$. The absorption wavelength corresponding to the particles formed under dark conditions (around $530 \mathrm{~nm}$ ) showed a variation of approximately $10 \mathrm{~nm}$ with respect to the ones formed in daylight $(520 \mathrm{~nm})$. Similarly, for the same reaction $\left(\mathrm{Au}^{3+}\right.$ salt $(1 \mathrm{mM})$ and $\mathrm{AS}$ extract $(1 \mathrm{~g} / 100$ $\mathrm{mL}$ ) in $1: 2$ volume ratio), there is an observable change in the SPR bands when the reaction conditions (such as sonication, heat treatment and exposure to sunlight) are changed. Additionally, the Av-AuNPs synthesized with microwave heat treatment $(280 \mathrm{~W} ; 20 \mathrm{~s})$, heating mantle $\left(50{ }^{\circ} \mathrm{C} ; 10 \mathrm{~min}\right)$, sonication (99\% power ratio; $20 \mathrm{~min}$ ) and under sunlight (20 $\mathrm{min}$ ) showed almost same absorbance wavelength (approx. $528 \mathrm{~nm}$ ) accompanied with a difference in the broadness of the peak. Comparing all the above mentioned conditions/variables for the preparation of the Au NPs, the SPR band is found to achieve the lowest wavelength around $520 \mathrm{~nm}$ under normal daylight. In general, the SPR band red shifts ${ }^{22,23}$ indicate the size increment of the formed NPs, which was found in the present study at higher $\mathrm{Au}^{3+}$ ion/AS extract concentrations as well as the reaction conditions imparting the kinetic energy to the reactants.

Furthermore, we synthesized Av-AuNPs of different sizes by varying the $\mathrm{pH}$ of avocado seed extract. The $\mathrm{pH}$ of the avocado seed extract is seen to play a vital role to control the morphology and size of the Au nanoparticles. Fig. 1 presents the UV-visible optical absorption spectra of the Av-AuNPs synthesized at different $\mathrm{pH}$ values of the reaction mixtures by avocado extract reduction (Fig. 1a) and their normalized absorption spectra (Fig. 1b). Formation of small sized Av-AuNPs is very clear from the appearance of characteristic surface plasmon resonance
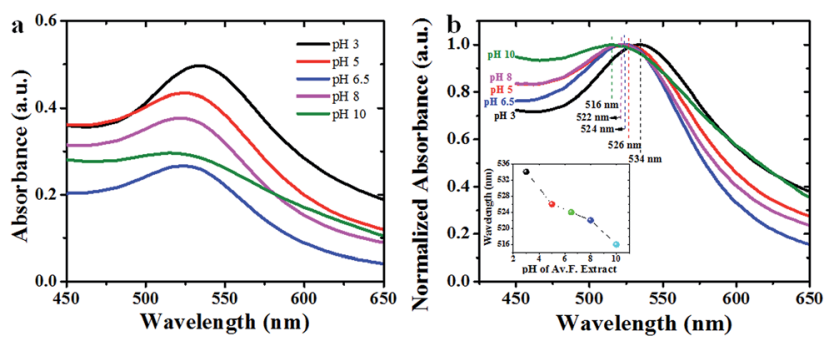

Fig. 1 (a) UV-visible absorbance spectra and corresponding (b) normalized absorbance studies of as-prepared colloidal Av-AuNPs at different $\mathrm{pH}$ values of the reaction mixtures (inset shows the $\mathrm{pH}$ values of avocado seed extract vs. wavelength from absorbance studies).
(SPR) bands in respective absorption spectra. While the position of the SPR band in the synthesized Av-AuNPs varied in systematic manner with the increase of reaction $\mathrm{pH}$. However, on average, the AuNPs fabricated using avocado seed extract reduction process are substantially smaller than previously reported coffee arabica fruit extract and Turkevich counterparts. ${ }^{23}$ Therefore, these samples were analyzed further in transmission electron microscope.

As can be noticed from the TEM images presented in Fig. 2, all the fabricated samples contain well-dispersed AuNPs. While the as-prepared NPs are of non-spherical shape, containing multiple facets, with occasional presence of twin-boundaries. Noteworthy, the morphology and shape of the NPs strongly depends on the $\mathrm{pH}$ of the avocado seed extract. The fabricated Av-AuNPs are abundant anisotropic with irregular shapes, including spherical NPs. While at $\mathrm{pH}=3$ (Fig. 2a) of avocado seed extract solution produces bigger particles maybe due to the limited accessibility of stabilizing agent (hydroxyl groups), at $\mathrm{pH}=10$ produces quasi-spherical NPs with smaller sizes (Fig. 2e). From the TEM images specify that it is quite possible to control the shape anisotropy and size of the nanoparticles using avocado seed extract mediated by controlling the $\mathrm{pH}$ of the green reducing and stabilizing agent. The possible effect of avocado seed extract-controlled $\mathrm{pH}$ values on the size control of the AuNPs has been represented schematically in the supplementary information Fig. S3. $\dagger$

The estimated size distributions of the AuNPs measured with DLS presented in Fig. $2 \mathrm{f}$ indicate the average sizes are very far to the values estimated from their DLS study, this may be due to the presence of biomass surrounded by the nanoparticle. Furthermore, to know the surface charge of the as-prepared AuNPs, zeta potential studies were carried out (Fig. 2f), which reveals the surface potential of the NPs is increasing with the lower to higher $\mathrm{pH}$ values. From these studies we can observe the low surface potential of AuNPs fabricated at $\mathrm{pH}=3$, due to low potential in the surface of the NPs there is a chance to agglomerate, which coincide with TEM images. Similarly, at $\mathrm{pH}$ $=10$ there is higher zeta potential which signifies the higher stability of as-prepared AuNPs.

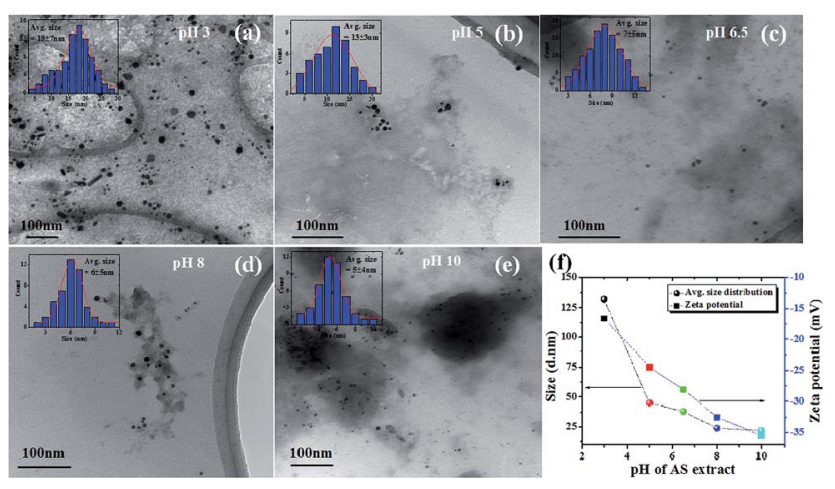

Fig. 2 (a-e) TEM images, and ( $f$ ) average size distributions and their corresponding zeta potential values of Av-AuNPs at $\mathrm{pH} 3,5,6.5,8$ and 10 respectively (inset shows the corresponding $\mathrm{pH}$ value size distribution analysis). 
The crystallinity of the as-prepared Av-AuNPs synthesized using avocado seed extract and their powder X-ray diffraction (XRD) patterns are shown in Fig. 3 where the diffraction patterns revealed sharp and well-defined peaks around 38.4, $44.6,64.8,77.8$ and $81.9^{\circ}$, corresponding to (111), (200), (220), (311), and (222) lattice planes of metallic gold in fcc phase (JCPDS \# 04-0783). The crystallinity and average grain sizes of Av-AuNPs were estimated using Debye-Scherer's relationship $D$ $=0.9 \lambda / \beta \cos \theta$ (where $D$ is the average grain size, $\theta$ is the diffraction angle, and $\beta$ is the full width at half maximum in radian) on the most intense (111) reflection of the NPs $(D \approx 12$ $\mathrm{nm})$.

The FTIR spectra of the avocado seed extract (Fig. 4a) reveals the characteristic bands located around $3322 \mathrm{~cm}^{-1}, 2932 \mathrm{~cm}^{-1}$, $1639 \mathrm{~cm}^{-1}, 1362 \mathrm{~cm}^{-1}, 1015 \mathrm{~cm}^{-1}$ and $924 \mathrm{~cm}^{-1}$ corresponding to the $-\mathrm{OH}$ stretching band, ${ }^{23,24} \mathrm{H}-\mathrm{C}-\mathrm{H}$ symmetric stretch alkanes, ${ }^{23} \mathrm{~N}=\mathrm{O}$ asymmetric stretching (nitrate), ${ }^{23} \mathrm{C}-\mathrm{N}$ stretching $^{24}$ and $\mathrm{C}-\mathrm{C}-\mathrm{N}$ asymmetric ${ }^{24}$ and symmetric bending, ${ }^{24}$ respectively. The corresponding bands have been attributed to the presence of phytochemicals in the AS extract. ${ }^{23,24}$ Similarly, FTIR bands of as-prepared Av-AuNPs show the presence of all bands common to the AS extract except a new broad band formation at 2300-2382 $\mathrm{cm}^{-1}$ (Fig. 4b) which may correspond to C-O stretching band. Moreover, the bands at 2932 and $924 \mathrm{~cm}^{-1}$ (Fig. 4a) are found to disappear after the formation of NPs. The appearance of the bands in the Av-NPs, with 1015 and $1154 \mathrm{~cm}^{-1}$ as peak intensity, were decreased as compared to AS extract peaks, which suggests that the functional groups corresponding to these bands might responsible for the reduction of $\mathrm{Au}^{3+}$ ions to AuNPs. The band at $1154 \mathrm{~cm}^{-1}$ corresponds to the $\mathrm{N}-\mathrm{X}$ stretching $\left(\mathrm{X}=\mathrm{NH}_{2}\right)$ suggest the presence of these phytochemicals on the surface of the synthesized Av-AuNPs. ${ }^{24,25}$

\section{Sensing and catalytic activity studies}

Sensing of $\mathbf{H g}$ (II) and $\mathbf{F e}($ III). For detection of metal ions, different salt solutions, such as $\mathrm{Cu}$ (II), $\mathrm{As}$ (III), $\mathrm{Fe}$ (II)/Fe(III), $\mathrm{Hg}$ (II), $\mathrm{Ni}$ (II) and $\mathrm{Co}$ (II), when added to a fixed volume of as-prepared AuNPs, a decrease in the absorbance intensity of AuNPs (Fig. S4 and S5 $\dagger$ ) is observed in almost all metal ions except

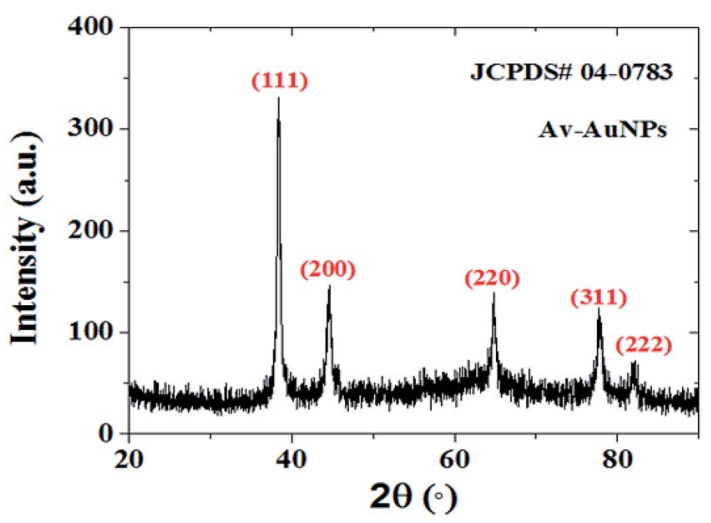

Fig. 3 XRD pattern of as-prepared Av-AuNPs using $1: 2$ volumetric ratio of $\mathrm{Au}^{3+}$ salt $\left(10^{-3}\right)$ and avocado seed extract $(1 \mathrm{~g} / 100 \mathrm{~mL})$.

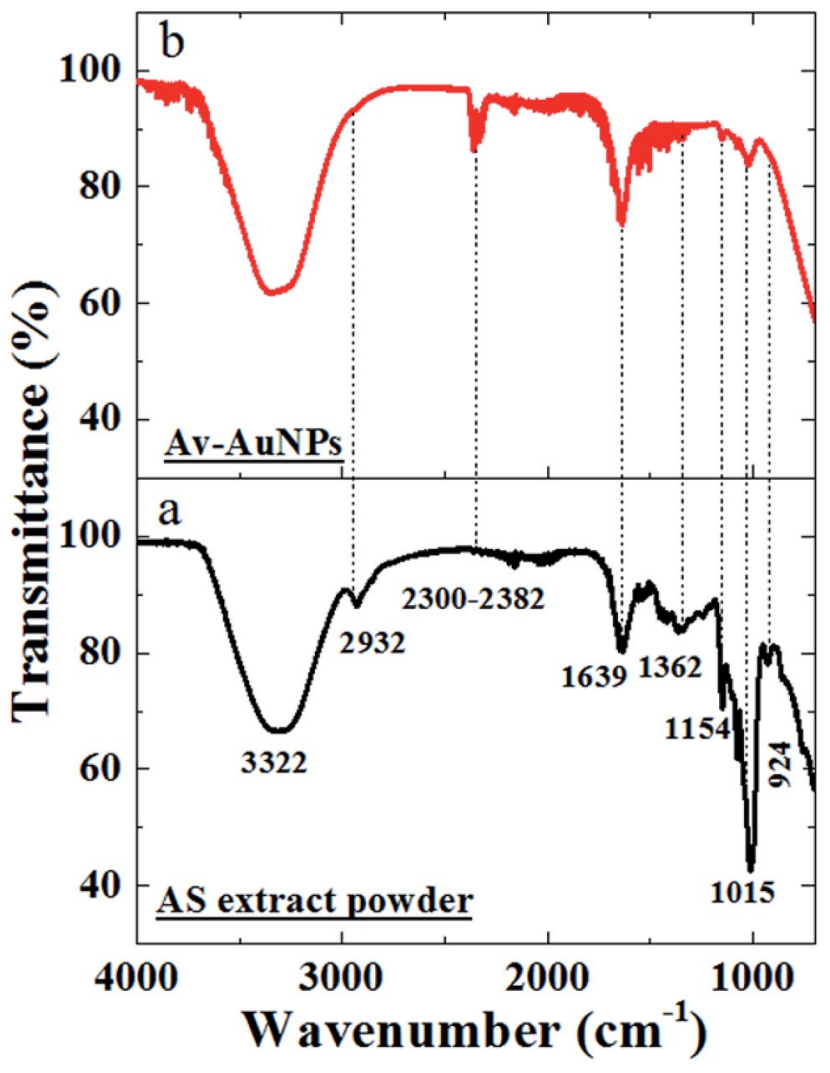

Fig. 4 FTIR spectra (through ATR accessory) of (a) avocado seed extract powder and (b) Av-AuNPs powder.

Fe(II). This implies that synthesized AuNPs possess weak selectivity toward detection of individual metal ions. This could be attributed to the presence of flavonoids and polyphenolic compounds, such as pyrogallol, catechol, gallic acid and their derivatives, in the biomass which are known to form complexes with heavy metal ions. ${ }^{20,25}$

Furthermore, to verify the selectivity of synthesized AuNPs towards heavy metal ions with subsequent addition of two metal ions was performed using UV-visible spectrometry. Fig. 5 shows the optical response of AuNPs in the presence of $\mathrm{Hg}$ (II) followed by the addition of $\mathrm{Fe}(\mathrm{III})$ and vice versa. The addition of $\mathrm{Hg}$ (II) to AuNPs-Fe(III) complex results in a blue shift of SPR peak, corresponding to AuNPs, from 519 to $509 \mathrm{~nm}$. The mechanism of $\mathrm{Hg}$ (II)/Fe(III) sensing in the presence of AuNPs$\mathrm{Fe}(\mathrm{III}) / \mathrm{Hg}$ (II) complex could be explained on the basis of Karthiga and Anthony report. ${ }^{21}$ The work reports ${ }^{21}$ the detection of $\mathrm{Hg}^{+2}$ using neem bark-silver nanoparticles (yellow colored) revealing a visual color change with the addition of $\mathrm{Hg}(\mathrm{II})(\mu \mathrm{M})$ i.e., complete decolorization of the nanoparticles with no observable change in the absorbance studies.

In previous reports related to the colorimetric detection of $\mathrm{Hg}$ (II) using $\mathrm{Au} \mathrm{NPs,} \mathrm{the} \mathrm{addition} \mathrm{of} \mathrm{Hg}$ (II) has been found to decrease the corresponding absorbance intensity ${ }^{\mathbf{2 1}}$ or the decrease in the absorbance intensity has been found to be accompanied with the red shift, implying the agglomeration of gold colloids in the presence of $\mathrm{Hg}$ (II). ${ }^{21}$ On contrary in present 

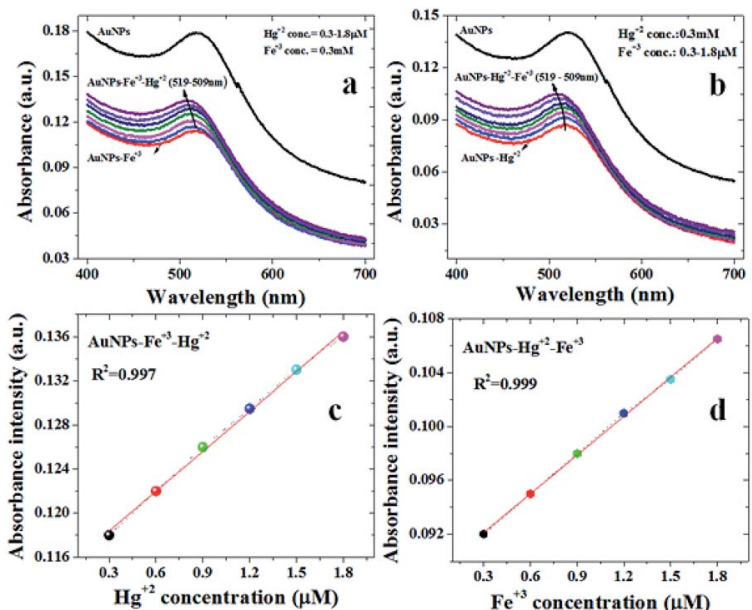

Fig. 5 Absorbance spectra for (a) the sensing of Hg(॥)using Av-AuNPs$\mathrm{Fe}(\mathrm{II})$ and (b) sensing of Fe(III) using Av-AuNPs-Hg(II); the linear fitting of concentration vs. absorbance intensity of (c) $\mathrm{Hg}(॥)\left(R^{2}=0.997\right)$ and (d) Fe(॥) sensing $\left(R^{2}=0.999\right)$.

study, a blue shift with an increase in the absorbance intensity of AuNPs is observed on increasing the concentration of $\mathrm{Hg}$ (II) in Fe(III) spiked distilled water. This may be due to an enhanced adsorption of $\mathrm{Hg}$ (II) on the surface of gold colloids in the presence of $\mathrm{Fe}(\mathrm{III})$ and forming a core shell structure. ${ }^{26,27}$ This kind of core shell structure may help to avoid the aggregation of gold colloids and form irrespective of the order of addition of $\mathrm{Fe}(\mathrm{III})$ and $\mathrm{Hg}$ (II) (Scheme 1).

The absorption amplitudes are found to increase with increasing concentration of $\mathrm{Hg}$ (II)/Fe(III). Accordingly, the summarized response shown in Fig. $5 \mathrm{c}$ and d, depicts the linear dependence of the absorption intensities with corresponding concentrations. It is worthwhile to contemplate that we observed a good linear relationship up to a concentration of 1.8 $\mu \mathrm{M}$ where a linear fitting of observed values reveals almost similar regression values of approx. 0.99 for $\mathrm{Hg}$ (II) and $\mathrm{Fe}$ (III) [see Fig. $5 \mathrm{c}$ and d]. Using the already reported evaluation criteria

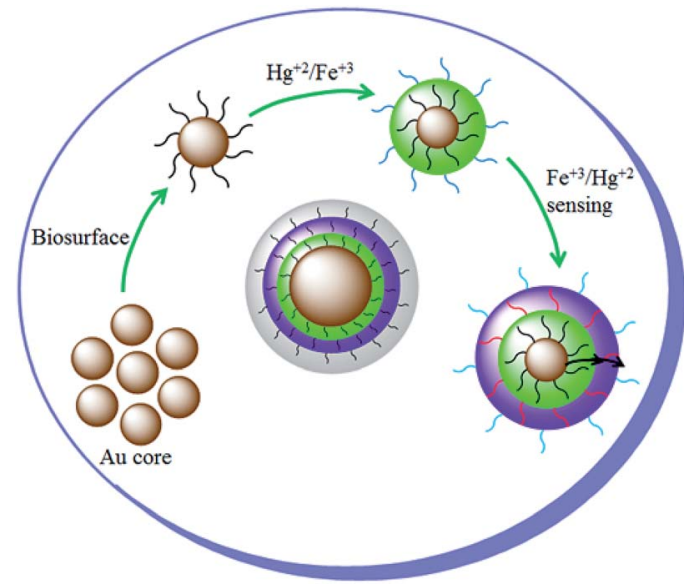

Scheme 1 Possible mechanism for $\mathrm{Hg}(॥)$ and Fe(III) sensing using AvAuNPs. (limit of detection $=3 \sigma / m$; where $\sigma$ and $m$ are the standard deviation and slope of the linear fit), ${ }^{28,29}$ the limit of detection is found to be $50 \mathrm{nM}$ and $30 \mathrm{nM}$ for $\mathrm{Hg}$ (II) and $\mathrm{Fe}(\mathrm{III})$ respectively. The comparison of the measured values (detection limit) with the already reported $\mathrm{Hg}$ (II) sensing in the literature (using AuNPs) is given in Table 1.

Catalytic activity of Av-AuNPs. On the other hand, industrial organic pollutants/dyes (4-NP, MB, MO, DB24, Rh6G and BrPB) are readily soluble in water. ${ }^{36,37}$ The initial addition of $\mathrm{NaBH}_{4}$ alone to the organic dyes failed to degrade any of the pollutants, but the addition of the proposed AuNPs, was found to initiate the degradation process of the pollutants (Table 2).

\section{Reduction of 4-nitrophenol (4-NP)}

4-nitrophenol is the crucial raw material for several chemical industries and it was extensively used in the manufacture industries such as pharmaceutical, pesticides, dyes, processing of leather and explosives. ${ }^{38}$ The serious breath or digestion of 4 NP can cause methemoglobinemia, fervescence, liver and kidney damage, and headaches etc.,.$^{38}$ Therefore, 4-NP has been listed as "priority pollutant" by the U. S. Environmental Protection Agency (EPA). ${ }^{37}$ The catalytic performance of asprepared gold nanoparticles was explored through the reduction of one of the most toxic pollutants 4-nitrophenol, with $\mathrm{NaBH}_{4}$ as the reducing agent (Fig. 6). The reaction process was monitored by a UV-vis absorbance spectrophotometer in successive time intervals. The 4-nitrophenol solution absorption peak was centered around $316 \mathrm{~nm}$ and absorption peak maxima was shifted from $316 \mathrm{~nm}$ to $400 \mathrm{~nm}$ due to the formation of its intermediate form (4-nitrophenolate ion) after the addition of $\mathrm{NaBH}_{4}$. The reaction did not proceed further (due to the absence of AuNPs) when monitored for a period of more than $20 \mathrm{~min}$. The addition of AuNPs to the solution of 4nitrophenolate ions results in the drastic decrement in the absorbance intensity (ref. to absorption peak maxima at $400 \mathrm{~nm}$ in Fig. 6a). The reduction of 4-nitrophenol could be regarded as a pseudo-first order reaction. As shown in Fig. 6b, linear relationship of $\ln \left(C_{t} / C_{0}\right)$ versus reaction time was observed, representing that the reduction of 4 -NP followed the pseudo-first order kinetics. The rate constant $k$ was calculated to be $1.55 \times$ $10^{-3} \mathrm{~s}^{-1}$ (Fig. 6c).

Table 1 Comparison of this methods for $\mathrm{Hg}^{2+}$ with other recently reported gold nanoparticle-based sensors ${ }^{a}$

\begin{tabular}{|c|c|c|c|c|}
\hline Sensing probe & Transducer & Linear range $(\mu \mathrm{M})$ & LOD (nM) & Ref. \\
\hline $\begin{array}{l}\text { Pyridine-rhodamine } \\
\text { conjugate probe }\end{array}$ & Fluorescent & $0-10$ & 630 & 30 \\
\hline DNA-AuNPs & SPR & $0.3-10$ & 300 & 31 \\
\hline MPA-AuNPs & Colorimetry & $0.2-0.5$ & 100 & 32 \\
\hline MPA/AMP-AuNPs & Colorimetry & $0.5-3.5$ & 500 & 33 \\
\hline R6G/MPA/AMP-AuNPs & FRET & $0.05-1$ & 50 & 34 \\
\hline Copolymer vesicle & Colorimetric & $0-10$ & 53 & 35 \\
\hline Av-AuNPs & SPR & $0.3-1.8$ & 50 & P.S. \\
\hline
\end{tabular}

${ }^{a}$ P.S. $=$ present study. 
Table 2 Catalytic efficiency of Av-AuNPs, size, reaction time and rate constant for the reduction of 4-NP and degradation of MB, MO, DB24, $\mathrm{BrPB}$ and Rh6G respectively ${ }^{a}$

\begin{tabular}{|c|c|c|c|c|c|}
\hline Organic pollutant/dyes & Catalyst & Size (nm) & Reaction time (sec) & $k\left(\mathrm{~s}^{-1}\right)$ & Ref. \\
\hline \multirow[t]{5}{*}{ 4-NP } & Au@NiAg & 10 & 270 & 0.0266 & 41 \\
\hline & $\mathrm{Au} /$ graphene hydrogel & 14.6 & 720 & 0.0032 & 42 \\
\hline & $\mathrm{Au} @ \mathrm{Fe}_{3} \mathrm{O}_{4}$ yolk shell & 15 & 300 & 0.0155 & 44 \\
\hline & Sr-AuNPs & 16 & 240 & $0.5098 \mathrm{~min}^{-1}$ & 45 \\
\hline & CA-AuNPs & $22 \pm 16$ & 2280 & $0.0522 \min ^{-1}$ & 23 \\
\hline \multirow[t]{5}{*}{ MB } & CA-AuNPs & $22 \pm 16$ & 840 & $0.052 \min ^{-1}$ & 23 \\
\hline & Sr-AuNPs & 16 & 3600 ( $\leq 50 \%$ degradation $)$ & 0.0118 & 45 \\
\hline & Fv-AuNPs & $\approx 20$ & 1380 & $0.0529 \mathrm{~min}^{-1}$ & 46 \\
\hline & SA-AuNPs & 26 & 720 & $0.0431 \mathrm{~min}^{-1}$ & 39 \\
\hline & Av-AuNPs & $8 \pm 5$ & 120 & $1.92 \times 10^{-3}$ & P.S. \\
\hline MO & DC-AuNPs & $\approx 10.5$ & 600 & $1.7 \times 10^{-3}$ & 47 \\
\hline & Av-AuNPs & $8 \pm 5$ & 60 & $8.5 \times 10^{-3}$ & P.S. \\
\hline \multirow[t]{2}{*}{$\mathrm{BrPB}$} & - & - & - & - & - \\
\hline & Av-AuNPs & $8 \pm 5$ & 120 & $0.32 \times 10^{-3}$ & P.S. \\
\hline \multirow[t]{2}{*}{ Rh6G } & - & - & - & - & - \\
\hline & Av-AuNPs & $8 \pm 5$ & 180 & $3.1 \times 10^{-3}$ & P.S. \\
\hline
\end{tabular}

\section{Degradation of methylene blue (MB)}

Methylene blue is a heterocyclic aromatic dye which has been widely using in the textile industries. ${ }^{31} \mathrm{Fig}$. $7 \mathrm{a}-\mathrm{c}$ provides the time-dependent UV-visible absorbance spectra of methylene blue catalyzed by as-prepared AuNPs. As a control/reference, the degradation process was monitored without AuNPs, i.e., the absorbance peak corresponding to $\mathrm{NaBH}_{4}+\mathrm{MB}$ solution,

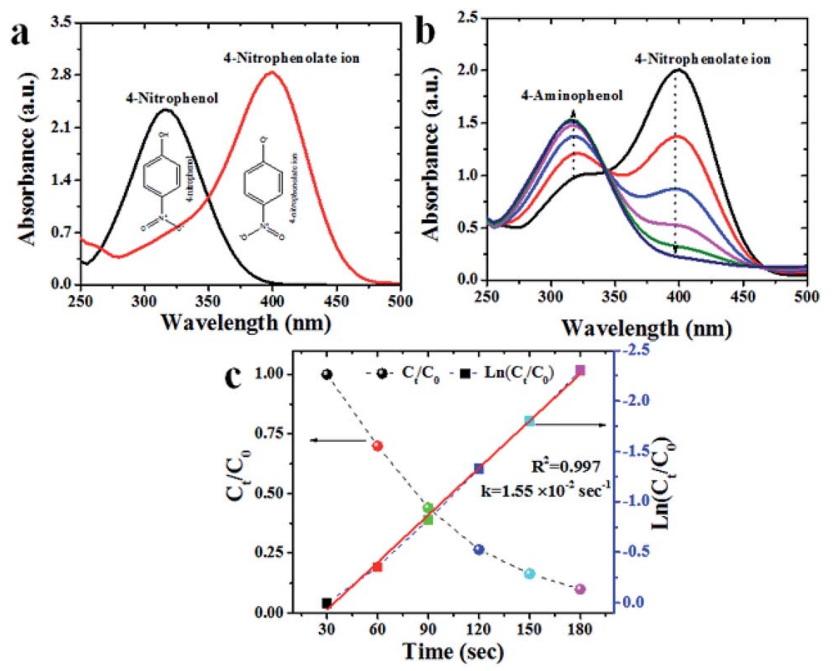

Fig. 6 Absorbance spectra for 4-nitrophenol (4-NP) with (a) $\mathrm{NaBH}_{4}$, (b) 4-NP $+\mathrm{NaBH}_{4}+\mathrm{Av}$-AuNPs and (c) kinetic study using first order pseudo reaction $\left(C_{t}\right.$ and $C_{0}$ corresponding to absorbance intensity at time $t$ and initial absorbance peak intensity respectively (rate constant $\left(k=1.55 \times 10^{-2} \mathrm{~s}^{-1}\right)$; regression coefficient $\left.\left.\left(R^{2}=0.997\right)\right)\right)$. reveals the characteristic absorbance of $\mathrm{MB}$ around 664 $\mathrm{nm}^{17,33,39,40}$ with no change in absorbance peak even after 20 min. After the addition of AuNPs, the absorbance peak rapidly decreased and finally disappeared within $120 \mathrm{~s}$, confirming the formation of Leucomethylene blue (more than $95 \%$ degradation). Hence representing the catalytic degradation of MB to LMB without by-product. As the primary concentration of $\mathrm{NaBH}_{4}$ was taken in excess and its concentration was considered as stable all over the reaction. Therefore, the degradation rate was assumed to be independent of $\mathrm{NaBH}_{4}$ concentration, and pseudo first-order kinetic equation could be applied to evaluate the catalytic rate (Fig. $7 \mathrm{~b}$ and $\mathrm{c}$ ). The absorbance of $\mathrm{MB}$ was proportional to only its concentration in the medium. The rate constant $k$ corresponding to the catalytic reduction was calculated as $1.92 \times 10^{-3} \mathrm{~s}^{-1}$.

\section{Degradation of methyl orange (MO)}

Methyl orange is an organic sulfosalt dye has been extensively used as an indicator in various fields. The excessive usage of this dye leads to several environmental and health hazards. ${ }^{38}$ Degradation of MO (concentration of $1 \mathrm{mM}$ ) was tested under ambient conditions (Fig. 7d-f). Pure MO had an absorbance peak maximum $\left(\lambda_{\max }\right)$ at $460 \mathrm{~nm}^{33}$. Although addition of reducing agent $\left(\mathrm{NaBH}_{4}\right)$ to MO solution brought no significant change in color or the absorbance peak (Fig. 7d), the addition of Av-AuNPs catalyst after $20 \mathrm{~min}$ (to the intermediate form of $\mathrm{MO}^{28}$ ) reveals a gradual decrease in the absorbance peak intensity with time (Fig. 7e). The complete disappearance of the peak (associated to the color change from orange to colorless) after $120 \mathrm{~s}$ of treatment is understood as $100 \%$ degradation of 

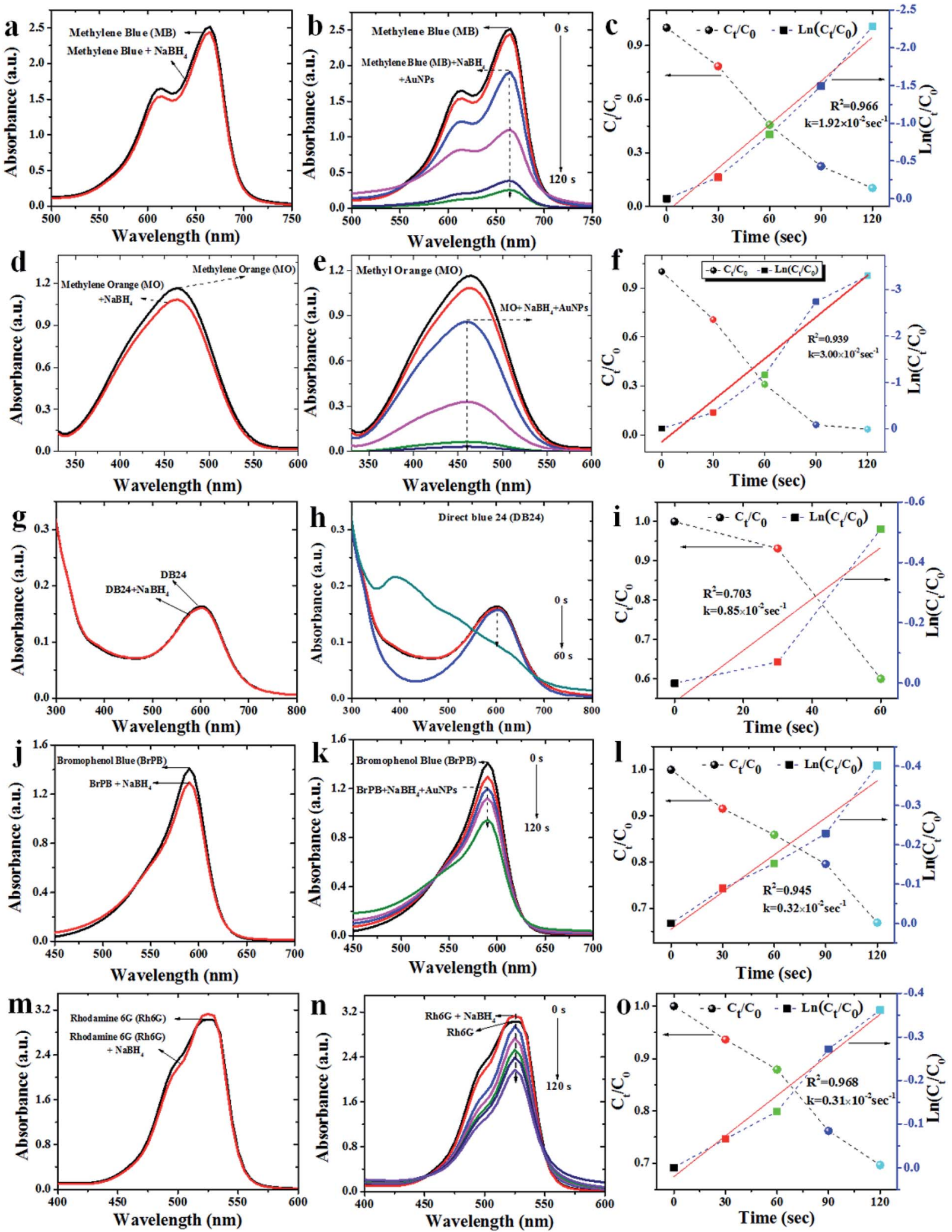

Fig. 7 Absorbance spectra for ( $a, d, g, j$ and $m$ ) Methylene blue (MB), Methyl orange (MO), Direct blue 24 (DB24), Bromophenol blue (BrPB) and Rhodamine 6G (Rh6G) with $\mathrm{NaBH}_{4}$, (b, e, h, k and n) presence of Av-AuNPs and (c, f, i, l and o) kinetic study using first-order pseudo reaction ( $C_{t}$ and $C_{0}$ corresponding to absorbance intensity at time $t$ and initial absorbance peak intensity respectively). 
the dye. The MO degradation rate was found to be linear as a function of time (Fig. 7f). The Av-AuNPs are found to maintain a very higher catalytic efficiency at a higher MO concentration in the solution. As the concentration of $\mathrm{NaBH}_{4}$ was very high it followed pseudo-first-order kinetics with $k=3 \times 10^{-2} \mathrm{~s}^{-1}$ (Fig. 7f).

\section{Degradation of direct blue (DB24)}

Direct blue 24 has been broadly used in leather, textile, plastic, paper and printing industries. Due to its non-degradability, it causes several environmental problems. ${ }^{39}$ For the catalytic degradation of DB24, using reducing agent $\mathrm{NaBH}_{4}$ and AuNPs, the absorbance peak $\left(\lambda_{\max }\right)$ of DB24 at $602 \mathrm{~nm}$ (Fig. 7g-i) was monitored. Similar to the above-mentioned pollutants, the addition of the reducing agent $\left(\mathrm{NaBH}_{4}\right)$ to $\mathrm{DB} 24$ (without AuNPs), no change in reaction was observed even after $20 \mathrm{~min}$. The addition of AuNPs to DB24 $+\mathrm{NaBH}_{4}$ solution gradually showed a decrease in the absorbance peak, confirming the complete catalytic degradation of DB24 (100\%) within $60 \mathrm{~s}$ (Fig. 7h). As the concentration of $\mathrm{NaBH}_{4}$ was very high, the decrease in the absorbance corresponding to DB24 was considered as a measure of the pseudo-first-order kinetic reaction with $k=0.8 .5 \times 10^{-3} \mathrm{~s}^{-1}$ (Fig. 7i).

\section{Degradation of bromophenol blue (BrPB)}

For the catalytic degradation of BrPB using reducing agent $\mathrm{NaBH}_{4}$ and AuNPs as catalyst, the absorbance peak $\left(\lambda_{\text {max }}\right)$ of BrPB around $590 \mathrm{~nm}$ (Fig. 7j) was monitored. As compared with the previous cases (4NP, MB and DB24), the addition of reducing agent $\left(\mathrm{NaBH}_{4}\right)$ to $\mathrm{BrPB}$ results in the slight reduction $(<5 \%)$ in the absorption intensity (observed after $20 \mathrm{~min}$ ) due to the high concentration of the reducing agent. Addition of the catalyst to $\mathrm{BrPB}+\mathrm{NaBH}_{4}$ solution revealed a gradual decrease of absorbance peak and the catalytic degradation of BrPB was found to complete $(\approx 40 \%$ ) within $120 \mathrm{~s}$ (Fig. 7k) following a pseudo-first-order reaction with $k=0.32 \times 10^{-3} \mathrm{~s}^{-1}$ (Fig. 7l).

\section{Degradation of rhodamine 6G (Rh6G)}

The usage of Rhodamine $6 \mathrm{G}$ is a (fluorescent organic dye) in textile and food industries has been increased in the past few years. It is proved to be highly carcinogenic and neurotoxic to living beings. ${ }^{40}$ For the catalytic degradation of Rh6G using reducing agent $\mathrm{NaBH}_{4}$ and AuNPs, the broad absorbance peak $\left(\lambda_{\max }\right)$ of Rh6G around $530 \mathrm{~nm}$ (Fig. 7m), was monitored. Contrary to the observation corresponding to the other pollutants, the addition of the reducing agent $\left(\mathrm{NaBH}_{4}\right)$ to $\mathrm{Rh} 6 \mathrm{G}$, an increment in the absorbance peak intensity $(20 \mathrm{~min})$ was observed. The addition of the catalyst to $\mathrm{Rh} 6 \mathrm{G}+\mathrm{NaBH}_{4}$ solution, revealed a gradual decrease of absorbance peak and the catalytic degradation of Rh6G was complete $(\approx 40 \%)$ within $180 \mathrm{~s}$ (Fig. 7n). As the concentration of $\mathrm{NaBH}_{4}$ was very high, the decrease corresponding to the absorbance peak reduction followed pseudo-first-order kinetics with $k=3.1 \times 10^{-3} \mathrm{~s}^{-1}$ (Fig. 7o).

In the present study as-prepared AuNPs acted as an efficient electron transfer mediator between Av-AuNPs and industrial organic pollutants and dyes as a redox catalyst. Moreover, AvAuNPs acted as a redox catalyst in the degradation/reduction of organic pollutants and dyes by electron relay effect between donor and acceptor molecules. Various reports ${ }^{23,39,41-48}$ are available on catalytic activity of gold nanoparticles for the reduction of major organic pollutant 4-NP and degradation of organic dyes MB, MO and DB24. From the reports (Table 2), it is clear that as the size of the gold nanoparticle decreases the required time span for the reduction or degradation of organic pollutant or dyes also decreases with increasing catalytic efficiency.

\section{Conclusions}

Conclusively, we have reported the fabrication of small gold nanoparticles (Av-AuNPs) using avocado seed extract at ambient conditions, sustaining the green chemistry principles. The concentration of avocado seed extract, gold solution, $\mathrm{pH}$ and external environmental conditions play an important role in controlling the surface plasmon resonance and size of the AvAuNPs. The sensing of $\mathrm{Hg}$ (II), employing green synthesis mediated small Av-AuNPs, has been successfully tested in the presence of $\mathrm{Fe}^{3+}$ in aqueous medium. As compared to the other reported metal ions, Av-Au nanoparticles reveal an excellent absorbance based selectivity towards $\mathrm{Hg}$ (II)/Fe(III), which has been explained using the formation of core-shell structure. Using UV-visible spectrometry (absorption spectra), the limit of detection is found to be $50 \mathrm{nM}$ and $30 \mathrm{nM}$ for $\mathrm{Hg}$ (II) and $\mathrm{Fe}$ (III) respectively. Moreover, an excellent catalytic efficiency of synthesized Av-AuNPs has been demonstrated for the reduction of contaminants such as 4-nitrophenol and degradation of several organic pollutants such as methylene blue (MB), methyl orange (MO), direct blue 24 (DB24), bromophenol blue (BrPB) and rhodamine blue (Rh6B). The corresponding kinetic studies revealed that the reduction and degradation process followed pseudo-first order kinetics model with less reaction time and more rate constant values. The catalytic efficiency of Av-AuNPs were monitored by UV-Vis spectra before and after the degradation of organic pollutant and dyes. The results show that AvAuNPs were also demonstrated to possess $100 \%$ catalytic reduction of 4-NP and degradation efficiency towards MB, MO and DB24.

\section{Conflicts of interest}

The authors declare no conflict of interest.

\section{Acknowledgements}

We thank to Dr Lorenzo Martinez Gomez, and Jose Juan RamosHernandez of ICF, UNAM for the support in the XRD analysis.

\section{References}

1 K. Manoj and P. Avinash, Indian J. Occup. Health, 2012, 16, 40-44. 
2 K. Baljeet, K. Navneet and K. Subodh, Coord. Chem. Rev., 2018, 358, 13-69.

3 M. Annadhasan, T. Muthu kumarasamy vel, B. V. R. Sankar and N. Rajendiran, ACS Sustainable Chem. Eng., 2014, 2, 887-896.

4 E. Priyadarshini and N. Pradhan, Sens. Actuators, B, 2017, 238, 888-902.

5 M. Rajkumar, T. Soundappan and C. Shen-Ming, Int. J. Electrochem. Sci., 2011, 6, 3164-3177.

6 N. Yogarajah and S. H. T. Scott, Environ. Sci.: Water Res. Technol., 2015, 1, 426-447.

7 C. Wang and Y. Chenxu, Rev. Anal. Chem., 2013, 32, 1-14.

8 K. Saha, S. A. Sarit, K. C. L. I. Xiaoning and M. R. Vincent, Chem. Rev., 2012, 112, 2739-2779.

9 M. R. Bindhu and M. Umadevi, J. Cluster Sci., 2013, 25, 969978.

10 G. Liu, M. Lu, X. Huang, T. Li and D. Xu, Sensors, 2018, 18, 4166.

11 K. Wu, B. Yang, X. Zhu, W. Chen, X. Luo, Z. Liu, X. Zhang and Q. Liu, New J. Chem., 2018, 42, 18749.

12 N. K. R. Bogireddy, V. Barba and V. Agarwal, ACS Omega, 2019, 4, 10702-10713.

13 K. Wu, X. Zhao, M. Chen, H. Zhang, Z. Liu, X. Zhang, X. Zhu and Q. Liu, New J. Chem., 2018, 42, 9578.

14 N. K. R. Bogireddy, U. Pal, M. K. Kumar, J. M. Domínguez, L. M. Gomez and V. Agarwal, Catal. Today, 2019, DOI: 10.1016/j.cattod.2019.06.044.

15 Y. Gao, K. Wu, H. Li, W. Chen, M. Fu, K. Yue, X. Zhu and Q. Liu, Sens. Actuators, B, 2018, 273, 1635-1639.

16 H. A. K. Kumar, B. K. Mandal, K. M. Kumar, S. B. Maddinedi, T. S. Kumar, P. Madhiyazhagan and A. R. Ghosh, Spectrochim. Acta, Part A, 2014, 130, 13-18.

17 N. K. R. Bogireddy, L. M. Gomez, I. O. Roman and V. Agarwal, Adv. Nano Res., 2017, 5, 253-260.

18 H. I. Eman, M. A. S. Aliyah, A. Eman, N. Arshi and M. O. Rawda, Int. J. Mol. Sci., 2018, 19, 2612.

19 R. C. Neelika, J. C. Allison, Z. Peter and V. Krasimir, Nanomaterials, 2018, 8, 496.

20 C. Yu, J. Tang, X. Liu, X. Ren, M. Zhen and L. Wang, Materials, 2019, 12, 189.

21 D. Karthiga and A. S. Philip, RSC Adv., 2013, 3, 16765.

22 L. Stephan and A. Mostafa, J. Phys. Chem. B, 1999, 103, 42124217.

23 N. K. R. Bogireddy, U. Pal, L. M. Gomez and V. Agarwal, RSC Adv., 2018, 8, 24819-24829.

24 S. Kannan, Int. J. Curr. Microbiol. Appl. Sci., 2014, 3, 341-351.
25 S. Gunasekharan, E. Sailatha, S. Seshadri and S. Kumaresan, Indian J. Pure Appl. Phys., 2009, 47, 12-18.

26 C. Radhakumary and K. Sreenivasan, Analyst, 2011, 136, 2959-2962.

27 T. Morris, H. Copeland, E. McLinden, S. Wilson and G. Szulczewski, Langmuir, 2002, 18, 7261-7264.

28 D. A. Melissa and D. M. Shelley, Electrode Potentials, in Hand book of electrochemistry, ed. C. G. Zoski, Elsevier science, U.S., 1st edn, 2007, pp. 813-827.

29 B. S. Baruah and R. Biswas, Sens. Actuators, B, 2018, 276, 8994.

30 T. Rasheed, F. Nabeel, C. Li and M. Bilal, J. Lumin., 2019, 208, 519-526.

31 L. Wang, T. Li, Y. Du, C. Chen, B. Li, M. Zhou and S. Dong, Biosens. Bioelectron., 2010, 25, 2622-2626.

32 C. C. Huang and H. T. Chang, Chem. Commun., 2007, 12, 1215-1217.

33 C. J. Yu and W. L. Tseng, Langmuir, 2008, 24, 12717-12722. 34 T. Rasheed, C. Li, F. Nabeel, W. Huang and Y. Zhou, Chem. Eng. J., 2019, 358, 101-109.

35 C. Umamaheswari, A. Lakshmanan and N. S. Nagarajan, J. Photochem. Photobiol., B, 2018, 178, 33-39.

36 P. S. Silva, B. C. Gasparini, H. A. Magosso and A. Spinelli, J. Hazard. Mater., 2014, 273, 70-77.

37 Y. Yang, M. He, M. Li, Y. Huang, T. Chi and Z. Wang, Inorg. Chem. Commun., 2018, 94, 5-9.

38 C. Fu, M. Li, C. Li and B. Yang, J. Alloy. Comp., 2017, 692, 727-733.

39 N. K. R. Bogireddy, K. K. H. Anand and B. K. Mandal, J. Mol. Liq., 2015, 211, 868-875.

40 U. Shanker, Environ. Chem. Lett., 2017, 15, 623-642.

41 S. Kulkarni, M. Jadhav, P. Raikar, S. Raikar and U. Raikar, Ind. Eng. Chem. Res., 2019, 58, 3630-3639.

42 J. Li, C. Liu and Y. Liu, J. Mater. Chem., 2012, 22, 8426.

43 X. Q. Wu, X. W. Wu, Q. Huang, J. S. Shen and H. W. Zhang, Appl. Surf. Sci., 2015, 331, 210.

44 F. Lin and R. Doong, J. Phys. Chem. C, 2017, 121, 7844.

45 I. Kumar, M. Mondal, V. Meyappan and N. Sakthivel, Mater. Res. Bull., 2019, 117, 18-27.

46 S. Balaji and M. B. Kumar, Adv. Powder Technol., 2017, 28, 785-797.

47 C. Umamaheswari, A. Lakshmanan and N. S. Nagarajan, J. Photochem. Photobiol., B, 2018, 178, 33-39.

48 M. Meena Kumari and D. Philip, Spectrochim. Acta, Part A, 2015, 135, 632-638. 